\title{
Diagnostic Accuracy of Computed Tomography-guided biopsy in
}

\section{pathological fractures}

1. Dr Christopher M. Stokes MBBS, BMedSci, PGDipSurgAnat (Orthopaedic

Registrar - Department of Orthopaedics, St Vincent's Hospital, Melbourne)

2. Dr Osama Elsewaisy MBBS (Orthopaedic Registrar - Department of Orthopaedics, St Vincent's Hospital, Melbourne)

3.Mr Grant Pang MBBS, FRACS, FAOrthA (Orthopaedic Surgeon - Department of Orthopaedics, St Vincent's Hospital Melbourne, Bone and Soft Tissue Sarcoma service, Peter MacCallum Cancer Centre, Melbourne, Victoria, Australia)

4. A/Prof John L. Slavin MBBS, FRCPA (Pathologist - Department of Pathology, St Vincent's Hospital Melbourne, Bone and Soft Tissue Sarcoma service, Peter MacCallum Cancer Centre, Melbourne, Victoria, Australia)

5. A/Prof Stephen M. Schlicht MBBS, FRANZCR (Radiologist - Director of Medical Imaging, St Vincent's Hospital, Melbourne, Bone and Soft Tissue Sarcoma service, Peter MacCallum Cancer Centre, Melbourne, Victoria, Australia)

6. Professor Peter F.M. Choong MBBS, MD, FRACS (Orthopaedic Surgeon -

This is the author manuscript accepted for publication and has undergone full peer review but has not been through the copyediting, typesetting, pagination and proofreading process, which may lead to differences between this version and the Version of Record. Please cite this article as doi: $10.1111 /$ ans.13894

This article is protected by copyright. All rights reserved. 
Director of Orthopaedics, St Vincent's Hospital Melbourne, Bone and Soft Tissue Sarcoma Service, Peter MacCallum Cancer Centre, Melbourne, Victoria, Australia, University of Melbourne, Department of Surgery)

An earlier version of this study was presented by Dr Christopher Stokes at the Australian Orthopaedic Association $75^{\text {th }}$ Annual Scientific Meeting, Brisbane, Queensland, on $13^{\text {th }}$ October 2015.

Abstract word count: 247

Word count: 2229 (excluding abstract, figure legends and references).

Manuscript contains 3 tables and 1 figure

Corresponding author:

Christopher M Stokes

41 Victoria Pde

Fitzroy VIC 3065

christopher.m.stokes@gmail.com

$+61392312211$

This article is protected by copyright. All rights reserved. 


\section{$\underline{\text { Keywords }}$}

pathological fracture; musculoskeletal tumour; biopsy; CT-guided biopsy; open biopsy

\section{$\underline{\text { Abstract }}$}

Introduction: Obtaining a histological diagnosis is essential for appropriate management of pathological fractures. Computed tomography (CT) is an accurate method of obtaining diagnosis for musculoskeletal tumours. We analysed whether diagnostic accuracy was maintained in the evaluation of pathological fractures.

Methods: A retrospective review of 101 consecutive patients presenting to our tertiary musculoskeletal tumour centre with pathologic fracture was performed. Patients underwent core needle biopsy under CT guidance of pathological fractures diagnosed by plain radiography and either CT or magnetic resonance imaging (MRI). The histopathology of the CT-guided biopsy was compared with the sample obtained from open biopsy or definitive surgery to determine diagnostic accuracy.

Results: The mean age at diagnosis was $52+/-20$ years (range 18-85) in a cohort of 46 men and 55 women. Diagnostic accuracy of CT-guided biopsy was $82.18 \%$. There were 65 malignant and 36 benign tumours with diagnostic accuracy of $86.15 \%$ and $80.56 \%$ respectively. The positive predictive value for a malignant tumour was $98.21 \%$ whilst it was $93.1 \%$ for benign tumours. The femur (53 cases) and humerus (25 cases) were the commonest bones fractured. The most frequent diagnoses were metastasis (20.79\%), giant cell tumour (17.82\%), osteosarcoma (9.90\%) and myeloma 
(9.90\%). There were no complications of CT-guided biopsy.

Conclusion: Pathologic fracture does not confound the diagnosis of musculoskeletal tumours. CT-guided biopsy is an accurate diagnostic tool in the evaluation of pathological fractures. Final diagnosis and management should be made in the context of appropriate anatomical and functional imaging using a multidisciplinary approach.

Word count: 247

\section{$\underline{\text { Introduction }}$}

Accurate diagnosis of bone and soft tissue tumours is vital and is used in conjunction with anatomical and functional imaging to guide management. Biopsy of pathological bone and soft tissue tumours is a critical step in their evaluation and management ${ }^{1}$. The goal of biopsy is to obtain diagnostic tissue whilst minimizing morbidity, limiting tumour spread and avoiding interference with future surgical management ${ }^{1,2}$. Although open biopsy is considered the gold standard, percutaneous core needle biopsy has been shown to yield similar results, with reported diagnostic accuracy ranging from 74 to 100 percent. $^{2-11}$

In instances where bone tumours present with a pathological fracture, image guided biopsy is increasingly employed to obtain tissue for histopathological analysis; however, the diagnostic accuracy has not been established and there are concerns that 
the diagnosis may be confounded by the physiologic changes in bone associated with fracture. Only one study analysed the accuracy of image guided biopsy in the setting of pathological fracture ${ }^{12}$. We hypothesized that the physiological responses to fracture such as haematoma and osteogenesis may affect the result of a biopsy. Therefore, the aim of this study was to determine whether the presence of fracture reduces the diagnostic accuracy of percutaneous CT-guided biopsy in the diagnosis of pathological bone tumours.

\section{Methods:}

St. Vincent's Hospital, Melbourne, is a major tertiary referral centre for musculoskeletal tumours and has a systematic process for the diagnosis of bone and soft tissue tumours. A CT-guided biopsy is often the final step in determining the diagnosis of pathological fractures. The decision to obtain a CT-guided biopsy is tailored to each individual patient, after review of their clinical presentation, anatomical and functional imaging, and local and systemic staging by bone and soft tissue tumour surgeons, radiologists, pathologists and oncologists in a weekly multidisciplinary team (MDT) meeting. After consideration by the MDT, select patients with multiple bony metastases in the setting of known metastatic disease may proceed to surgical stabilization without prior biopsy.

After obtaining ethical review board approval (QA: 001/16), we performed a 
retrospective review of 101 patients who presented to our institution with a pathological fracture; had undergone a CT-guided core needle biopsy; and subsequently had a surgical procedure where operative specimens were obtained. This took the form of either a dedicated surgical biopsy to obtain a diagnostic specimen (open biopsy) or a surgical procedure to treat the fracture, at which intraoperative samples were taken for histopathology (excisional biopsy). We compared the histology obtained by CT-guided biopsy to the final histology obtained at surgery and hence determined the diagnostic accuracy of the CT-guided biopsy.

Patient details were retrieved from the St. Vincent's Hospital Bone and Soft Tissue Tumour Registry in January 2016, including data from January 1997 to December 2015. The histology results were obtained from the hospital pathology database. Patients who had undergone CT-guided biopsy but not open biopsy or surgical management of the lesion were excluded from the study. Similarly, patients who underwent surgical management of pathological fractures without prior biopsy were excluded.

The core needle biopsy of bone lesions at St. Vincent's Hospital is performed in the radiology department under $\mathrm{CT}$-guidance. The biopsy is taken using aseptic technique, with intravenous analgesia and conscious sedation. Dense cortical lesions are biopsied using a $12 \mathrm{G}$ or $13 \mathrm{G}$ biopsy needle with an Arrow OnControl Powered Bone Access Driver (Teleflex, Morrisville, North Carolina, USA). Biopsies adjacent 
to neurovascular structures that require more control are performed using the Bonopty needle (AprioMed, Uppsala, Sweden). Generally 1-3 samples are taken via the same biopsy tract, depending on the quality of specimens obtained, and the proximity to neurovascular structures.

\section{Results:}

A total of 101 patients with pathological fractures diagnosed on imaging underwent CT-guided biopsy with subsequent open biopsy or surgical excision. The mean age at presentation was $52+/-19.71$ years (range 18-85) in a cohort of 46 men and 55 women. Eighty-three of the $101 \mathrm{CT}$-guided biopsy results were consistent with the final histopathology, producing a diagnostic accuracy of $82.18 \%$. There were no complications of CT-guided biopsy in our series.

Eighteen of the 101 CT-guided biopsy results did not correlate with final histopathology, including 10 malignant and eight benign diagnoses based on final pathology. Two benign CT-biopsy results of fibrous dysplasia (FD) and enchondroma turned out to be malignant fibrous histiocytoma (MFH) and low-grade chondrosarcoma respectively. One diagnosis of FD and one diagnosis of myeloma turned out to be aneurysmal bone cyst $(\mathrm{ABC})$ and fibrous tissue respectively. The remaining 14 cases were non-diagnostic showing only fracture callus, haematoma or necrosis. Open biopsy was used to obtain a diagnosis for 12 of these cases. The 
remaining two cases were metastases confirmed with histopathology obtained at definitive surgery (Table 1).

A total of 65 malignant tumours were diagnosed at final histopathology with a CTguided biopsy diagnostic accuracy of $86.15 \%$ (56/65). Fifty-five of the 56 tumours diagnosed as malignant by CT-guided biopsy were confirmed as malignant on surgical pathology. This produced a positive predictive value of $98.21 \%(55 / 56)$ for malignant tumours (Figure 1). Eight of the 14 inconclusive CT-guided biopsy results were later diagnosed as malignant. The most frequent malignant diagnoses were metastasis (21 cases), osteosarcoma (10), myeloma (10) and chondrosarcoma (9). A complete breakdown is displayed in Table 2 .

There were 36 benign diagnoses at final histopathology. The diagnostic accuracy of CT-guided biopsy for benign tumours was 80.56\% (29/36). Twenty-seven of the 29 benign tumours diagnosed with CT-guided biopsy correlated with final histopathology. This gave a positive predictive value of $93.10 \%(27 / 29)$ for benign tumours (Figure 1). Included was one diagnosis of reactive bony changes, initially classified as an inconclusive CT-guided biopsy result. The most common benign conditions were giant cell tumour (18 cases), enchondroma (6) and $\mathrm{ABC}$ (6) (see Table 2).

This article is protected by copyright. All rights reserved. 
The femur was the most common site of pathological fracture with 53 cases and an overall accuracy of $75.5 \%(40 / 53)$. The next most common sites were the humerus (25 cases) and tibia (9 cases) with diagnostic accuracies of $88 \%(22 / 25)$ and $100 \%$ (9/9) respectively. A complete breakdown of diagnostic accuracy according to fracture location is shown in Table 3.

Pathological fractures with extraosseous extension demonstrated a higher accuracy $(92.5 \%)$ than intraosseous lesions $(75.4 \%)$. Three of the 40 pathological fractures with extraosseous extension returned a non-diagnostic CT-guided biopsy result. In comparison, 15 of the 61 pathological fractures confined to bone returned a noncorrelated or inconclusive CT-guided biopsy result.

\section{Discussion:}

Biopsy is a fundamental component in the diagnosis of musculoskeletal tumours. Although open biopsy remains the gold standard, CT-guided percutaneous biopsy is an accepted alternative with diagnostic accuracy ranging from 74 to 100 percent. $^{2,3,10,12-14}$ Factors affecting diagnostic accuracy of CT-guided biopsy of bone tumours include anatomical location, extraosseous involvement and number of samples collected. ${ }^{3,12,15}$ Additionally, biopsies performed at a referring centre are six times more likely to be non-diagnostic than those at a tertiary institution. ${ }^{16,17}$ 
The treatment of pathologic fractures is challenging. The rate of nonunion has been reported to be up to 50 percent. Additional considerations may be concomitant chemotherapy or previously irradiated wounds. More importantly, it is vital that appropriate imaging and accurate tissue diagnosis is obtained in order to facilitate treatment that will prevent potential limb and life threatening complications. ${ }^{12,18}$

We hypothesized that the diagnostic accuracy of CT-guided biopsy may be reduced in the setting of pathological fracture due to the physiological processes at a fracture site such as haematoma, inflammation and osteogenesis. In our series, 11 of the 18 noncorrelated CT-guided biopsy results showed haematoma, fracture callus or fibrosclerotic bone.

In our review of the English language literature, Datir et al ${ }^{12}$ reported the only series that investigated the accuracy of imaging guided needle biopsy in pathological fractures. They analysed 129 imaging guided biopsies performed on patients with fractures through presumed pathological lesions. In their series, diagnostic samples (amenable to histological analysis) were obtained in $75.5 \%, 66 \%$ and $93.7 \%$ for $\mathrm{CT}$, fluoroscopy and US guided biopsy respectively; for an overall diagnostic yield of $77 \%$. Their diagnostic accuracy was $96 \%$ for patients in whom diagnostic samples were obtained. 
Our series also demonstrated CT-guided biopsy to be a safe and accurate means of determining diagnosis in pathological fractures. The overall accuracy of $82.18 \%$ is comparable to those of CT-guided biopsy results for musculoskeletal tumours in the absence of fracture. ${ }^{3,19,20}$ Datir et al reported a diagnostic accuracy of 97.3\% (72 of 74 cases); however, this pertains only to the cohort who had a diagnostic CT-guided biopsy. If the 16 non-diagnostic biopsy results in our series were excluded (see Fig. 1), 82 of 85 diagnostic samples correlated with final histopathology giving an accuracy of $96.5 \%$. We believe it is important to include non-diagnostic samples in calculating accuracy because of the clinical implications of an inconclusive biopsy result. Like Datir et al ${ }^{12}$ we do not report any complications of percutaneous biopsy in this series.

Percutaneous biopsy is a cost effective alternative to open biopsy for musculoskeletal tumours. $^{2,14,16,17}$ Traina et al calculated the cost of percutaneous biopsy at \$1106USD compared to between $\$ 4321.25$ USD and \$7234.00 USD for incisional biopsy. ${ }^{2}$ In addition to cost, reduced contamination rates and lower risk of complications $(0-10 \%$ percutaneous versus $<16 \%$ incisional biopsy ${ }^{17}$ ) are significant benefits. ${ }^{13,14,16,17,21}$

A missed diagnosis of malignancy can have catastrophic consequences, limit treatment options and compromise patient care. Although metastases and multiple myeloma are far more common causes of pathological fracture, ${ }^{12,22}$ failure to diagnose a primary bone sarcoma may result in otherwise avoidable amputation. ${ }^{17}$ Biopsy is a 
critical step in reaching the diagnosis. ${ }^{23}$ Importantly, $98.21 \%$ of malignant diagnoses made with CT-guided biopsy in this series correlated with final histopathology. This is not to underestimate a wrong diagnosis of malignancy with CT-biopsy and the unnecessary tests, interventions and stress imparted onto the individual and their family. However, in this series only one incorrect diagnosis of malignancy (multiple myeloma) occurred from CT-guided biopsy, which was later corrected to fibrous tissue.

Two benign CT-guided biopsy results (fibrous dysplasia and enchondroma) were later confirmed as malignant (malignant fibrous histiocytoma and low-grade chondrosarcoma respectively) at open biopsy. Histopathology from a CT-guided biopsy is reviewed at our multi-disciplinary meeting. Despite benign diagnoses in these instances, a significant level of doubt persisted on review of imaging and pathology. Subsequently, both cases proceeded to open biopsy, which provided the definitive diagnosis. This illustrates three key points: (1) the importance of a multidisciplinary approach at a tertiary centre for the diagnosis of musculoskeletal tumours, (2) biopsy results should be correlated with important diagnostic adjuncts such as clinical findings, anatomical and functional imaging and (3) open biopsy has an important diagnostic role and remains the gold standard. ${ }^{2}$

In their retrospective analysis, Datir et al reported higher accuracies for biopsies of tumours with extraosseous involvement versus those confined to bone. ${ }^{12}$ Our results 
compared favourably to Datir et al with a $92.5 \%$ accuracy for tumours with extraosseous extension versus $75.4 \%$ for solely intraosseous lesions. ${ }^{12}$ This is perhaps a result of our hypothesis that physiological processes occurring at a fracture site may obscure a biopsy result. Datir et al recommend consideration for immediate open biopsy in pathological fractures confined to bone. ${ }^{12}$ However, we believe that further evidence is required before making this recommendation, given the high accuracy of percutaneous biopsy along with the reduced complication rate and cost. ${ }^{2}$

Diagnostic accuracy may vary according to anatomical location. ${ }^{3}$ Other authors analysed diagnostic accuracy at different anatomical sites, with appendicular skeletal lesions having higher yield than pelvic or spine lesions. ${ }^{14}$ In this series, $77 \%$ of fractures involved the femur or humerus, which correlates to incidences reported in the literature. ${ }^{3,12}$ Subgroup analyses of anatomical locations were not performed due to the low numbers in remaining groups. Nevertheless, the diagnostic accuracy of femoral fractures $(75.5 \%)$ and humeral fractures $(88.8 \%)$ were consistent with overall results.

We acknowledge that this retrospective analysis has limitations. Data were entered into our Registry prospectively but were not recorded specifically for this analysis. Only patients who underwent open biopsy or surgical excision following CT-guided biopsy were included in the study. Reasons for not progressing to open biopsy or surgical excision included low clinical and radiological index of suspicion, patient 
preference and being medically unfit for further intervention. Additionally, there are a number of radiologists who performed the musculoskeletal biopsies. The number of diagnostic samples taken at biopsy varied at our institution with the interventionist, anatomical location and characteristics (e.g. vascular) of the lesion influencing the number of samples taken. It has been suggested that a minimum of three specimens should be obtained in bone lesions to optimize the diagnostic yield. ${ }^{15}$ However, this can be difficult depending on the size of the lesion and its proximity to neurovascular structures.

In conclusion, this series has shown that CT-guided biopsy is a safe and accurate means of determining diagnosis of pathological fractures - a topic with a paucity of literature. Further evidence in the form of well-powered, prospective, comparative studies would be of benefit to guide management by identifying factors associated with poor percutaneous biopsy results.

Our recommendation is that clinically suspected pathological fractures are appropriately investigated after careful history and examination. This typically involves anatomical and often functional imaging. Early referral to a tertiary musculoskeletal oncology centre is advised. Oncological principles should apply and CT-guided biopsy of such fractures should be performed at a tertiary musculoskeletal tumour centre. Biopsy route should be determined with consideration of excision of the biopsy tract at a multi-disciplinary meeting amongst oncological surgeons and 
radiologists performing the biopsy. Consideration is also to be given to surrounding structures and subsequent definitive surgery.

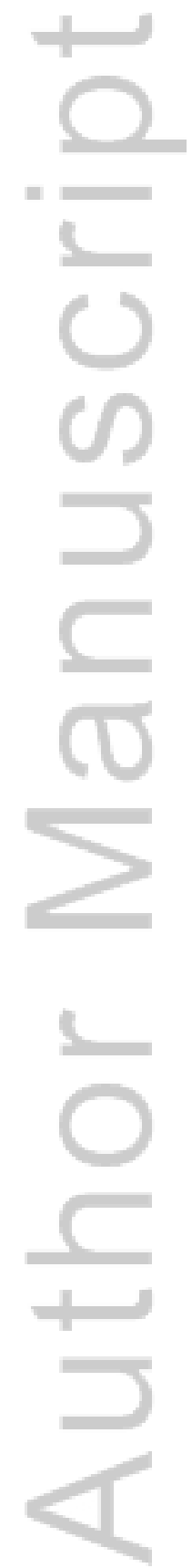

This article is protected by copyright. All rights reserved. 


\section{$\underline{\text { References: }}$}

1. Dickinson I; Duggal A; Choong P. Biopsy of Musculoskeletal Tumours. ANZ Journal of Surgery. 2004;74:2.

2. Traina F; Errani C; Toscano A; et al. Current Concepts in the Biopsy of Musculoskeletal Tumors. Journal of Bone and Joint Surgery. 2015;97(7):6.

3. Altuntas A; Slavin J; Smith P; et al. Accuracy of Computed Tomography Guided Core Needle Biopsy of Musculoskeletal Tumours. ANZ Journal of Surgery. 2005;75:5.

4. Seng C; Png W; Tan M. Accuracy of core needle biopsy for musculoskeletal tumours. Journal of Orthopaedic Surgery. 2013;21(1):4.

5. Stoker D; Cobb J; Pringle J. Needle biopsy of musculoskeletal lesions. A review of 208 procedures. J Bone Joint Surg. Br. 1991;73:498-500.

6. Heslin M; Lewis J; Woodruff J; Brennan M. Core needle biopsy for diagnosis of extremity soft tissue sarcoma. Ann. Surg. Oncol. 1997;4:425-31.

7. Ball A; Fisher C; Pittam M; Watkins R; Westbury G. Diagnosis of soft tissue tumours by Tru-Cut biopsy. Br. J. Surg. 1990;77:756-8.

8. Skrzynski M; Biermann J; Montag A; Simon M. Diagnostic accuracy and charge-savings of outpatient core needle biopsy compared with open biopsy of musculoskeletal tumors. J Bone Joint Surg Am. 1996;78(644-9).

This article is protected by copyright. All rights reserved. 
9. Ray-Coquard I; Ranchere-Vince D; Thiesse P; et al. Evaluation of core needle biopsy as a substitute to open biopsy in the diagnosis of soft-tissue masses. Eur J Cancer. 2003;39:2021-5.

10. Rimondi E; Staals E; Errani C; et al. Percutaneous CT-guided biopsy of the spine: results of 430 biopsies. Eur Spine J. 2008;17(7):975-81.

11. Issakov J; Flusser G; Kollender Y; Merimsky O; Lifschitz-Mercer B; Meller I. Computed tomography-guided core needle biopsy for bone and soft tissue tumors. Isr Med Assoc J. 2003;5(1):28-30.

12. Datir A; Pechon P; Saifuddin A. Imaging-Guided Percutaneous Biopsy of Pathologic Fractures: A Retrospective Analysis of 129 Cases. American Journal of Roentgenology. 2009;193:5.

13. Dupuy D; Rosenberg A; Punyaratabandhu T; Tan M; Mankin H. Accuracy of CT-guided needle biopsy of musculoskeletal neoplasms. AJR Am. J. Roentgenol. 1998;171:759.

14. Hau A; Kim I; Kattapuram S. Accuracy of CT-guided biopsies in 359 patients with musculoskeletal lesions. Skeletal Radiol. 2002;31:349-353.

15. Wu J; Goldsmith J; Horwich P; Shetty S; Hochman M. Bone and soft-tissue lesions: what factors affect diagnostic yield of image-guided core-needle biopsy? Radiology. 2008;248:8.

16. Mankin H; Lange T; Spanier S. The hazards of biopsy in patients with malignant primary bone and soft-tissue tumors. J Bone Joint Surg Am. 1982;64:1121-7.

This article is protected by copyright. All rights reserved. 
17. Mankin H; Makin C; Simon M. The hazards of the biopsy, revisited. J Bone Joint Surg Am. 1996;78(5):656-63.

18. Gainor B; Buchert P. Fracture healing in metastatic bone disease. Clin Orthop Relat Res. 1983;178:297-302.

19. Jelinek J; Murphey M; Welker J; et al. Diagnosis of primary bone tumors with image-guided percutaneous biopsy: Experience with 110 tumours. Radiology. 2002;223:731-7.

20. Welker J; Henshaw R; Jelinek J; Shmookler B; Malawer M. The percutaneous needle biopsy is safe and recommended in the diagnosis of musculoskeletal masses. Cancer. 2000;89:2677-86.

21. Yao L; Nelson S; Seeger L; Eckardt J; Eilber F. Primary musculoskeletal neuplasms: Effectiveness of core-needle biopsy. Radiology. 1999;212:682-6.

22. Sim F. Metastatic bone disease of the pelvis and femur. Instr Course Lect. $1992 ; 41: 317-327$.

23. Errani C; Traina F; Perna F; Calamelli C; Faldini C. Current concepts in the biopsy of musculoskeletal tumors. ScientificWorldJournal. 2013;2013:538152. Epub 2013 Jun 5.

This article is protected by copyright. All rights reserved. 


\section{Figure Legends:}

Figure 1: Inclusion flow chart of CT-guided biopsy patients with final pathology result.

Note: 1 patient with a benign CT-Guided Biopsy demonstrated no pathological lesion on open biopsy (only fracture callus)

Table 1: Non-correlated CT-guided biopsy results.

Table 2: Breakdown of final histopathology diagnoses.

Note: Of 2 patients with benign 'reactive bone changes', $1=$ fracture callus, $1=$ benign cyst

Table 3: Diagnostic accuracy according to anatomical location.

This article is protected by copyright. All rights reserved. 


\section{malignant $(n=56)$}

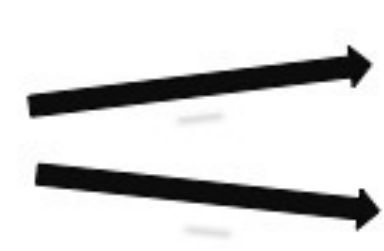

benign $(n=29)$

inconclusive

$$
(n=16)
$$

malignant $(n=55)$

benign $(n=1)$

benign $(n=27)$

malignant $(n=2)$

malignant $(n=8)$

benign $(n=8)$

\section{Slide1.jpg}


Table 1 Non-Correlated CT-Guided Biopsy Results

\begin{tabular}{|l|l|l|l|l|}
\hline Case & CT-biopsy & Open biopsy & Excisional biopsy & Location \\
\hline 1 & non-diagnostic & & metastasis & femur \\
2 & haematoma & & metastasis & humerus \\
3 & haematoma & enchondroma & & metatarsal \\
4 & haematoma & frozen section & MFH & femur \\
5 & haematoma & frozen section & ABC & femur \\
6 & haematoma & frozen section & ABC & distal femur \\
7 & haematoma & frozen section & fibrous dysplasia & femur \\
8 & haematoma & GCT & & distal radius \\
9 & fracture callus & myeloma & & distal femur \\
10 & fracture callus & myeloma & & humerus \\
11 & fracture callus & frozen section & ABC & femur \\
12 & fibrous dysplasia & MFH & & femur \\
13 & fibrous dysplasia & ABC & & distal femur \\
14 & enchondroma & Low-grade & & mid-shaft femur \\
& chondrosarcoma & & \\
15 & necrotic bone & frozen section & osteosarcoma & femur \\
16 & necrotic bone & frozen section & angiosarcoma & femur \\
17 & fibrosclerotic & High grade & & femur \\
& bone & pleomorphic sarcoma & & \\
18 & myeloma & & fibrous tissue & femur \\
\hline
\end{tabular}

${ }^{*}$ Proximal aspect of long bone unless otherwise stated

This article is protected by copyright. All rights reserved. 
Table 2 Breakdown of final histopathology diagnoses

\begin{tabular}{|lr|lr|}
\hline \multicolumn{4}{|c|}{ Final Diagnosis } \\
\hline \multicolumn{1}{|c|}{ Malignant } & \multicolumn{1}{c|}{ Benign } \\
\hline Chondrosarcoma & 9 & Aneurysmal Bone Cyst (ABC) & 6 \\
Osteosarcoma & 10 & Giant cell tumour (GCT) & 18 \\
Angiosarcoma & 1 & Fibrous dysplasia & 4 \\
Leiomyosarcoma & 1 & Enchondroma & 6 \\
Spindle cell sarcoma & 1 & Reactive bone changes & 2 \\
Malignant fibrous histiocytoma & & & \\
(MFH) & 4 & & \\
High-grade pleomorphic sarcoma & 3 & & \\
Myeloma & 10 & & \\
Lymphoma & 5 & & \\
Metastasis & 21 & & \\
& & & \\
\hline Total & 65 & Total & \\
\hline
\end{tabular}

This article is protected by copyright. All rights reserved. 
Table 3 Diagnostic accuracy according to anatomical location

\begin{tabular}{|lrr|}
\hline Anatomical Location & $\boldsymbol{n ( = 1 0 1 )}$ & Accuracy \\
\hline Femur & 53 & $75.5 \%$ \\
Humerus & 25 & $88 \%$ \\
Tibia & 9 & $100 \%$ \\
Foot & 5 & $80 \%$ \\
Radius & 4 & $75 \%$ \\
Pelvis & 2 & $100 \%$ \\
Ulna & 1 & $100 \%$ \\
Hand & 1 & $100 \%$ \\
\hline Clavicle & 1 & $100 \%$ \\
Rib & 1 & $100 \%$ \\
\hline Total & 101 & - \\
\hline
\end{tabular}

This article is protected by copyright. All rights reserved. 


\section{University Library}

\section{- M M N E R VA A gateway to Melbourne's research publications}

Minerva Access is the Institutional Repository of The University of Melbourne

Author/s:

Stokes, CM;Elsewaisy, O;Pang, G;Slavin, JL;Schlicht, SM;Choong, PFM

Title:

Diagnostic accuracy of computed tomography-guided biopsy in pathological fractures

Date:

2017-07-01

Citation:

Stokes, C. M., Elsewaisy, O., Pang, G., Slavin, J. L., Schlicht, S. M. \& Choong, P. F. M. (2017). Diagnostic accuracy of computed tomography-guided biopsy in pathological fractures. ANZ JOURNAL OF SURGERY, 87 (7-8), pp.600-604. https://doi.org/10.1111/ ans.13894.

Persistent Link:

http://hdl.handle.net/11343/292659 